\title{
A Novel Heterozygous ACAN Variant in a Short Patient Born Small for Gestational Age with Recurrent Patellar Dislocation: A Case Report
}

\author{
(D) Su Ji Kim, (D) Jong Seo Yoon, (D) II Tae Hwang \\ Hallym University Kangdong Sacred Heart Hospital, Hallym University College of Medicine, Department of Pediatrics, Seoul, Korea
}

\begin{abstract}
What is already known on this topic?
$A C A N$ is located on chromosome $15 q 26.1$ and variants in this gene impair chondrogenesis at the growth plate, resulting in defects in longitudinal bone growth and skeletal development. Heterozygous $A C A N$ variants can induce several clinical characteristics in patients, including variable degrees of short stature and mild skeletal dysplasia, such as midface hypoplasia, joint problems, brachydactyly, broad great toes, and lumbar lordosis.
\end{abstract}

\section{What this study adds?}

We report a Korean patient with a novel nonsense variant, c. 1968C > G, p.(Tyr656*), in ACAN. The patient was born small for gestational age (SGA) and presented with short stature and displayed several dysmorphic features including genu valgum, cubitus valgus, and recurrent patellar dislocations. Patellar dislocation is a rare clinical feature of patients with $A C A N$ defects. ACAN variants should be considered in short stature patients born SGA with joint problems, particularly those with recurrent patellar dislocation and genu valgum.

\begin{abstract}
ACAN variants can manifest as various clinical features, including short stature, advanced bone age (BA), and skeletal defects. Here, we report rare clinical manifestations of $A C A N$ defects in a 9 year, 5 month-old girl born small for gestational age (SGA), who presented with short stature, and was initially diagnosed with idiopathic growth hormone deficiency. She displayed several dysmorphic features, including genu valgum, cubitus valgus, and recurrent patellar dislocations. She presented with progressive advancement of BA compared with chronological age. Whole exome sequencing confirmed the presence of a novel heterozygous nonsense variant, C.1968C $>$ G, p.(Tyr656*), in ACAN. ACAN variants should be considered in short stature patients born SGA with joint problems, particularly those with recurrent patellar dislocation and genu valgum.
\end{abstract}

Keywords: $A C A N$, short stature, patellar dislocation, small for gestational age

\section{Introduction}

Aggrecan is a component of the extracellular matrix around the cartilage growth plate and provides a hydrated gel structure for the load-bearing properties of joints. The aggrecan gene, ACAN, is located on chromosome 15q26.1 and variants of this gene impair chondrogenesis at the growth plate, resulting in longitudinal bone growth and skeletal development defects (1). Heterozygous $A C A N$ variants can induce several clinical characteristics in patients, including variable degrees of short stature and mild skeletal dysplasia, such as midface hypoplasia, joint problems, brachydactyly, broad great toes, and lumbar lordosis $(2,3,4)$. Short patients with $A C A N$ variants often present with advanced bone age (BA), which is different from the short stature associated with endocrine diseases, such as idiopathic growth hormone deficiency (IGHD) and hypothyroidism, which are related to delayed BA. Therefore, advanced BA may lead clinicians to test specifically for $A C A N$ variants in patients with short stature and other clinical features, such as skeletal defects $(5,6)$. However, as $A C A N$ variant patients without advanced BA have also been reported, advanced BA may suggest 
the presence of $A C A N$ variant but cannot be referred to as a specific characteristic of $A C A N$ variants (7). In clinical practice, $A C A N$ variants are difficult to diagnose because their phenotype-genotype relationships are inconsistent.

Here, we report a case of a patient with a novel ACAN variant presenting with patellar dislocation and suggest that this rare clinical feature of $A C A N$ variant may be a definitive clue for diagnosis.

\section{Case Report}

A girl aged 9 years and 5 months was referred to a pediatric endocrinology clinic for evaluation when she presented with abnormally reduced stature. Her height was $115 \mathrm{~cm} \mathrm{[-3.93}$ Korean standard deviation score (SDS)] and weight $35.2 \mathrm{~kg}$ (0.60 SDS) (Figure 1A) (8). According to the Tanner stage for puberty, her breast development was at stage 3 and pubic hair development was at stage 1 . She was born at 40 weeks through normal vaginal delivery with birth weight $2800 \mathrm{~g}$ $\left(-1.53 \mathrm{SDS} ; \leq 10^{\text {th }}\right.$ percentile); she was diagnosed as small for gestational age (SGA). Her mother's height was $161 \mathrm{~cm}(-0.01$ SDS) and the father's height was $175 \mathrm{~cm}(0.10 \mathrm{SDS})$, yielding a predicted adult height - calculated using mid-parental height - of $161.5 \mathrm{~cm}$ (0.09 SDS) (Figure 1A). Family history also included short stature, with a recorded height of 150 $\mathrm{cm}(-2.36 \mathrm{SDS})$ in her paternal grandmother (Figure 1B). The subject exhibited various skeletal defects, including cubitus valgus, genu valgum, and recurrent patellar dislocation. BA was 10 years (1.37 SDS) and within the normal range when compared with her chronological age (CA). No abnormalities were identified in either chemistry or thyroid function and the karyotype of the subject was normal (46, XX). The levels of basal luteinizing hormone, follicle-stimulating hormone, and estradiol were $2.02 \mathrm{mIU} / \mathrm{mL}, 6.16 \mathrm{mIU} / \mathrm{mL}$, and $25 \mathrm{pg} /$ $\mathrm{mL}$, respectively, confirming that the pubertal stage. We performed growth hormone provocation tests using arginine and L-dopamine without sex steroid priming, and the peak levels were $0.29 \mathrm{ng} / \mathrm{mL}$ and $2.3 \mathrm{ng} / \mathrm{mL}$, respectively. No abnormal findings were identified using sellar magnetic resonance imaging and the patient was treated with 0.23 $\mathrm{mg} / \mathrm{kg} /$ day of recombinant human growth hormone (rhGH) to combat IGHD. One year after initiation of treatment, the age of the subject was 10 years and 6 months, and her height had increased to $124.1 \mathrm{~cm}(-2.97 \mathrm{SDS})$. Serum insulin-like growth factor-1 levels were in the upper half of the reference range (0.69 SDS) after rhGH treatment. BA was shown to be advanced by 1 year and 6 months (2.31 $\mathrm{SDS}$ ). Gonadotropin-releasing hormone agonist (GnRHa) treatment was added to the rhGH treatment to suppress bone maturation. She developed knee deformity over the previous four years, with recurrent intermittent pain during walking. She showed no improvement in her knee deformity and pain with conservative treatment. At the age of 10 years and 10 months, she underwent knee realignment surgery to correct a progressive bilateral genu valgum deformity and address her recurrent patellar dislocations.

DNA was extracted from EDTA blood samples and the entire exome was captured using SureSelectXT Human A11 Exon v5 (Agilent Technologies Inc., Santa Clara, CA, USA), followed by sequencing using NextSeq (Illumina Inc., San Diego, CA, USA). The reported variant was confirmed using Sanger

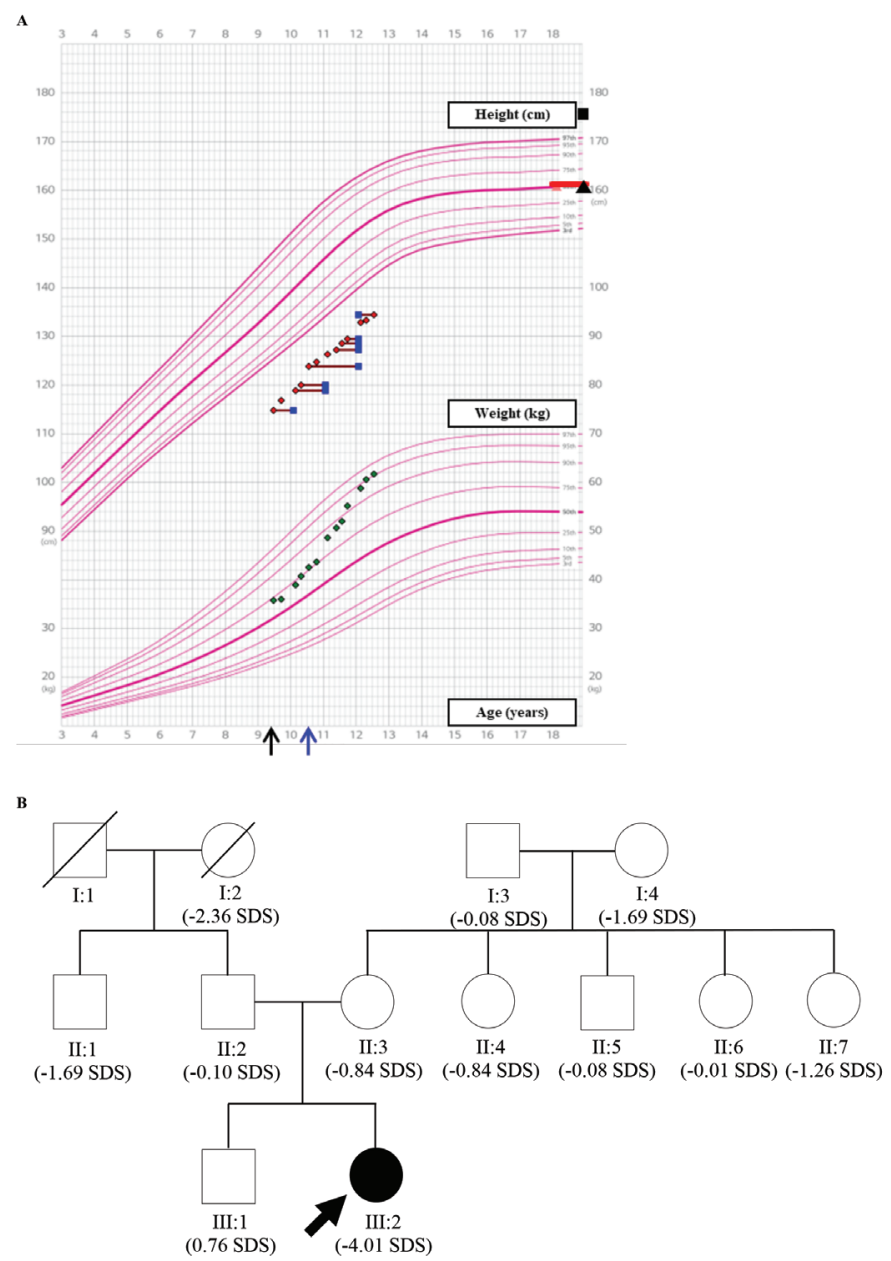

Figure 1. (A) Reference growth chart for Korean females (318 years). Heights and weights are shown as red and green dots, respectively. bone age is represented as blue dots. Black squares and triangles indicate the height of the father and the mother, respectively. Mid-parental height is shown as the red line. The time point at which the patient started treatment with recombinant human growth hormone is indicated by a black arrow, and the time point at which gonadotropin-releasing hormone agonist treatment was started is indicated by a blue arrow. (B) Pedigree of the patient. The patient is indicated by a black arrow; the height SDS corresponding to the age of each family member is also indicated. SDS: standard deviation score

SDS: standard deviation score 
sequencing. WES revealed a novel heterozygous variant, NM_013227.3:c.1968C > G, p. (Tyr656*), in exon 10 of ACAN (Figure 2). The pathogenicity of the variant was evaluated in accordance with the American College of Genetics and Genomics (9). This variant was considered pathogenic in accordance with the PSV1, PM2, and PP3 criteria, and was not found in the Genome Aggregation Database (gnomAD) or the Korean Reference Genome Database (KRGDB). Thus, we concluded that it represents a novel pathogenic variant that results in a nonsense variant of the tyrosine residue to a stop codon.

After 3 years and 6 months of rhGH treatment, the patient was receiving $\mathrm{rhGH}(0.3 \mathrm{mg} / \mathrm{kg} /$ day $)$ and her height was $137.9 \mathrm{~cm}(-2.91 \mathrm{SDS})$. She has since been rehabilitated and her walking has stabilized.

\section{Discussion}

In this report, we confirmed a novel heterozygous ACAN variant using WES. The subject presented with short stature, SGA, IGHD, progressive advanced BA, and various skeletal defects including cubitus valgus, genu valgum, and recurrent patellar dislocations.

The increase in genetic testing to determine the underlying cause of idiopathic short stature (ISS) has highlighted the importance of $A C A N$ variants. Recently, the $A C A N$ variant has been shown to be one of the most common causes of ISS with a prevalence of $1-6 \%(10,11)$. In addition, ACAN presents with autosomal dominant (AD) inheritance, i.e., ACAN variants are also a common cause of familial ISS (12).

Aggrecan is a major proteoglycan component of the extracellular matrix in joints and intervertebral disc cartilage and provides the hydrated gel structure necessary to facilitate the load-bearing properties of skeletal joints
(1). Therefore, aggrecanopathy resulting from $A C A N$ variant has been linked to several degrees of skeletal defects and presents with some consistent clinical characteristics, such as advanced BA and early growth cessation (13). However, $A C A N$ variants with genotype-phenotype correlation are not always consistent, resulting in a high degree of clinical heterogeneity.

Advanced BA is considered a hallmark of $A C A N$ variant but some patients develop delayed BA. ACAN variant with short stature and delayed BA was first reported in 2017 (7). A study showed that 79 out of 110 affected patients (72\%) experienced advanced BA, suggesting that accelerated skeletal maturation may be a useful indicator of $A C A N$ variant, but is insufficient as a clear clinical feature $(2,4,10)$. The initial BA of the subject was within the normal range compared to that for her $\mathrm{CA}$, but her $\mathrm{BA}$ progressed to more than 12 months in relation to $C A$ after one year of rhGH treatment. It can be assumed that IGHD influences the progression of BA. Previous studies have reported that GnRHa treatment was successfully applied to prevent bone aging $(2,4)$, as was also evident in this case.

It has been suggested that the possibility of $A C A N$ variant in short-statured patients born SGA should be considered (4). A flowchart to determine the possibility of having an ACAN variant was proposed by these authors. The clinical features of midface hypoplasia, joint problems, and broad great toes among short-statured patients born SGA were considered to be linked to the possibility of having $A C A N$ variant. In this earlier study, since only $13.8 \%$ of patients with $A C A N$ variant with advanced BA were found, the researchers reported that advanced BA was not sufficient as an indicator of $A C A N$ variant. Among the major clinical symptoms presented in the literature, that is midface hypoplasia, joint problems, and broad great toes, the subject of this case had only one characteristic feature, namely joint problems. According to

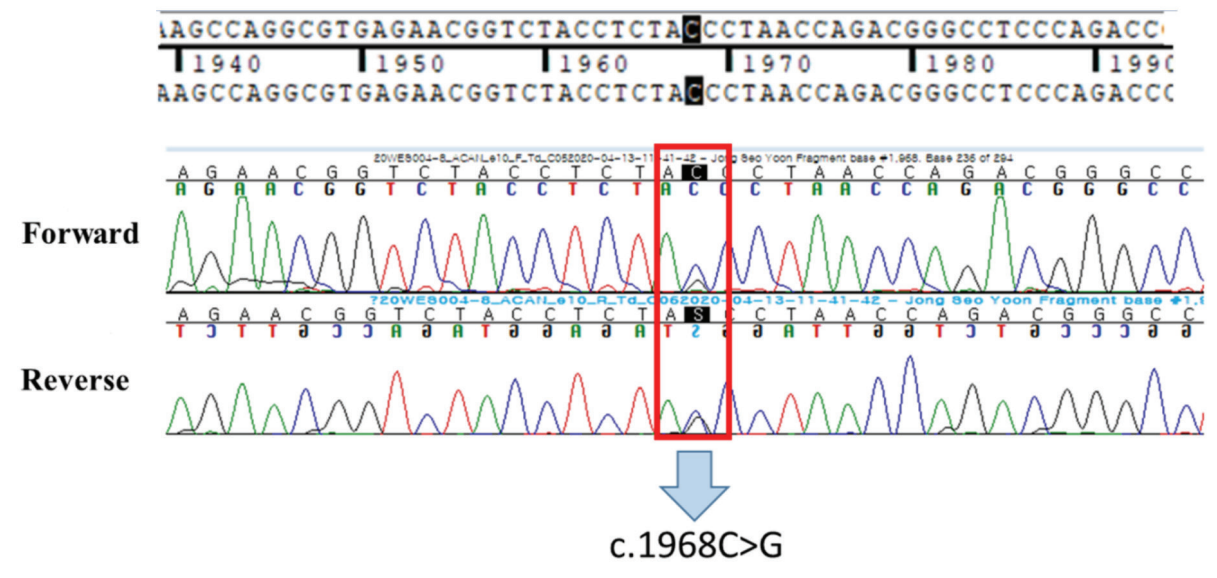

Figure 2. Results of $A C A N$ sanger sequencing in the patient

NM_013227.3:c.1968C > G, p.(Tyr656*), heterozygote, nonsense 
the proposed flowchart, the probability of $A C A N$ variant was rare in this subject.

Skeletal defects from ACAN variants diagnosed in ISS do not present with serious defects. Osteoarthritis is a representative feature of the joint problems associated with the ACAN variant; however, the subject in this case did not experience any osteoarthritis, but developed genu valgum and recurrent knee dislocations at a young age. In a previous study, knee dislocation was found to be a relatively rare clinical feature reported in only a few affected patients (3). Knee dislocation is a very rare symptom and considered a hallmark of suspected $A C A N$ variant when accompanied by short stature.

$A C A N$ variant is inherited in an $\mathrm{AD}$ fashion, but the parents of the subject were not short in stature. The grandmother of the subject was short in stature, but she grew up in a period of poor nutrition and hence environmental factors cannot be excluded. The parents of the subject did not want to undergo genetic testing, so we could not test them for genetic abnormalities. Owing to these limitations, we have assumed that the patient had a de novo heterozygous $A C A N$ variant.

\section{Conclusion}

We report a novel heterozygous variant of $A C A N$. If short statured children born SGA without catch-up growth exhibit joint problems, particularly recurrent patellar dislocation and genu valgum, we would suggest that these patients be immediately referred for genetic testing as there is a possibility that they may harbor an $A C A N$ variant.

\section{Ethics}

Informed Consent: A written informed consent was obtained from her parents.

Peer-review: Externally peer-reviewed.

\section{Authorship Contributions}

Surgical and Medical Practices: Il Tae Hwang, Concept: Su Ji Kim, Jong Seo Yoon, Il Tae Hwang, Design: Jong Seo Yoon, Il Tae Hwang, Data Collection or Processing: Su Ji Kim, Analysis or Interpretation: Su Ji Kim, Jong Seo Yoon, Il Tae Hwang, Literature Search: Su Ji Kim, Jong Seo Yoon, Il Tae Hwang, Writing: Su Ji Kim, Jong Seo Yoon, Il Tae Hwang.

Financial Disclosure: The authors declared that this study received no financial support.

\section{References}

1. Kiani C, Chen L, Wu YJ, Yee AJ, Yang BB. Structure and function of aggrecan. Cell Res 2002;12:19-32.
2. Nilsson O, Guo MH, Dunbar N, Popovic J, Flynn D, Jacobsen C, Lui JC, Hirschhorn JN, Baron J, Dauber A. Short stature, accelerated bone maturation, and early growth cessation due to heterozygous aggrecan mutations. J Clin Endocrinol Metab 2014;99:1510-1518. Epub 2014 Apr 24

3. Sentchordi-Montané L, Aza-Carmona M, Benito-Sanz S, Barreda-Bonis AC, Sánchez-Garre C, Prieto-Matos P, Ruiz-Ocaña P, Lechuga-Sancho A, Carcavilla-Urquí A, Mulero-Collantes I, Martos-Moreno GA, Del Pozo A, Vallespín E, Offiah A, Parrón-Pajares M, Dinis I, Sousa SB, Ros-Pérez P, González-Casado I, Heath KE. Heterozygous aggrecan variants are associated with short stature and brachydactyly: Description of 16 probands and a review of the literature. Clin Endocrinol (Oxf) 2018;88:820-829. Epub 2018 Mar 24

4. van der Steen M, Pfundt R, Maas SJWH, Bakker-van Waarde WM, Odink RJ, Hokken-Koelega ACS. ACAN Gene Mutations in Short Children Born SGA and Response to Growth Hormone Treatment. J Clin Endocrinol Metab 2017;102:1458-1467.

5. Baron J, Sävendahl L, De Luca F, Dauber A, Phillip M, Wit JM, Nilsson O. Short and tall stature: a new paradigm emerges. Nat Rev Endocrinol 2015;11:735-746. Epub 2015 Oct 6

6. Dateki S. ACAN mutations as a cause of familial short stature. Clin Pediatr Endocrinol 2017;26:119-125. Epub 2017 Jul 27

7. Tatsi C, Gkourogianni A, Mohnike K, DeArment D, Witchel S, Andrade AC, Markello TC, Baron J, Nilsson O, Jee YH. Aggrecan Mutations in Nonfamilial Short Stature and Short Stature Without Accelerated Skeletal Maturation. J Endocr Soc 2017;1:1006-1011.

8. Kim JH, Yun S, Hwang SS, Shim JO, Chae HW, Lee YJ, Lee JH, Kim SC, Lim D, Yang SW, Oh K, Moon JS; Committee for the Development of Growth Standards for Korean Children and Adolescents; Committee for School Health and Public Health Statistics, the Korean Pediatric Society; Division of Health and Nutrition Survey, Korea Centers for Disease Control and Prevention. The 2017 Korean National Growth Charts for children and adolescents: development, improvement, and prospects. Korean J Pediatr 2018;61:135-149. Epub 2018 May 28

9. Richards S, Aziz N, Bale S, Bick D, Das S, Gastier-Foster J, Grody WW, Hegde M, Lyon E, Spector E, Voelkerding K, Rehm HL; ACMG Laboratory Quality Assurance Committee. Standards and guidelines for the interpretation of sequence variants: a joint consensus recommendation of the American College of Medical Genetics and Genomics and the Association for Molecular Pathology. Genet Med 2015;17:405-424. Epub 2015 Mar 5

10. Hauer NN, Sticht H, Boppudi S, Büttner C, Kraus C, Trautmann U, Zenker M, Zweier C, Wiesener A, Jamra RA, Wieczorek D, Kelkel J, Jung AM, Uebe S, Ekici AB, Rohrer T, Reis A, Dörr HG, Thiel CT. Genetic screening confirms heterozygous mutations in ACAN as a major cause of idiopathic short stature. Sci Rep 2017;7:12225.

11. Plachy L, Strakova V, Elblova L, Obermannova B, Kolouskova S, Snajderova M, Zemkova D, Dusatkova P, Sumnik Z, Lebl J, Pruhova S. High Prevalence of Growth Plate Gene Variants in Children With Familial Short Stature Treated With GH. J Clin Endocrinol Metab 2019;104:4273-4281.

12. Stavber L, Hovnik T, Kotnik P, Lovrečić L, Kovač J, Tesovnik T, Bertok S, Dovč K, Debeljak M, Battelino T, Avbelj Stefanija M. High frequency of pathogenic ACAN variants including an intragenic deletion in selected individuals with short stature. Eur J Endocrinol 2020;182:243-253.

13. Kim TY, Jang KM, Keum CW, Oh SH, Chung WY. Identification of a heterozygous ACAN mutation in a 15-year-old boy with short stature who presented with advanced bone age: a case report and review of the literature. Ann Pediatr Endocrinol Metab 2020;25:272-276. Epub 2020 Jul 29 